\title{
Intraosseous vascular access in adults using the EZ-IO in an emergency department
}

\author{
Adeline Su-Yin Ngo • Jen Jen Oh • Yuming Chen • \\ David Yong • Marcus Eng Hock Ong
}

Received: 21 January 2009 / Accepted: 2 June 2009 /Published online: 11 August 2009

(C) Springer-Verlag London Ltd 2009

\begin{abstract}
Background Intraosseous (IO) access is an alternative to conventional intravenous access.

Aims We evaluate the use of the EZ-IO ${ }^{\mathrm{TM}}$ as an alternative vascular access for patients in the emergency department.

Methods A non-randomized, prospective, observational study was performed in adults using the EZ-IO ${ }^{\mathrm{TM}}$ powered drill device.

Results Twenty-four patients were recruited. There were 35 intraosseous insertions, including 24 tibial and 11 humeral insertions. All EZ-IOTM insertions were achieved within $20 \mathrm{~s}$ and were successful at the first attempt except for one. Of the intraosseous insertions, $88.6 \%$ were reported to be easier than intravenous cannulation. We found flow rates to be significantly faster using a pressure bag. The seniority of operators did not affect the success of insertion. Complications included a glove being caught in the drill device and extravasation of fluid although they were easily preventable. Conclusion The use of the EZ-IO ${ }^{\mathrm{TM}}$ provides a fast, easy and reliable alternative mode of venous access, especially in the resuscitation of patients with no venous vascular access in the emergency department. Flow rates may be improved by the use of pressure bags.
\end{abstract}

The views expressed in this paper are those of the author(s) and not those of the editors, editorial board or publisher.

A. S.-Y. Ngo $(\bowtie) \cdot$ J. J. Oh • D. Yong $\cdot$ M. E. H. Ong

Department of Emergency Medicine, Singapore General Hospital, Outram Road,

Singapore 169608, Singapore

e-mail: Adeline.ngo.s.y@sgh.com.sg

Y. Chen

Singapore Clinical Research Institute,

Singapore, Singapore
Keywords Intraosseous needle Intravenous access . Infusion rates

\section{Introduction}

The intraosseous (IO) route for vascular access was initially described in 1922 [1] and was used commonly during World War II [2]. Although the intravascular route is the standard for venous access in medical practice, IO administration of fluids and medications can be an alternative option when the conventional method of intravenous access fails.

Drugs administered intraosseously enter the circulation as fast and in the same concentration as those administered intravenously [3,4]. However, there have not been any published clinical trials assessing the use of the EZ-IO ${ }^{\mathrm{TM}}$ in the busy emergency department (ED) of a tertiary hospital setting although there have been studies on the paediatric population [3-11].

Intraosseous access has recently been revived in adults as an alternative when conventional intravenous access may be difficult or impossible [3, 12-15]. The standard IO needle has been compared with needles having a newer design and most recently with IO access devices [3, 11, 12, 16-19]. The EZ-IO ${ }^{\mathrm{TM}}$ is one such device for adult IO vascular access.

We carried out a non-randomized, prospective, observational study evaluating the use of the EZ-IO ${ }^{\mathrm{TM}}$ powered drill device for IO access in adults presenting to a local tertiary ED.

\section{Methods}

We conducted a non-randomized, prospective, observational study in adults using the EZ-IO ${ }^{\mathrm{TM}}$ powered drill device. 
This study was approved by our hospital Ethics Committee. Waiver of consent was obtained as the procedures were deemed to be life saving in the critically ill requiring peripheral access that would not have otherwise been obtained by traditional methods quickly. If the patient was awake enough to understand, verbal and if possible written informed consent was obtained from the patient.

The study population included patients who presented to the Department of Emergency Medicine of Singapore General Hospital (SGH), an urban hospital that is Singapore's oldest and largest acute tertiary hospital and national referral centre.

The SGH Department of Emergency Medicine handles nearly 120,000 patients annually, of which about $9 \%$ are resuscitations (priority 1), 33\% are major emergencies (priority 2), 56\% are ambulant/minor emergencies (priority 3 ) and $2 \%$ are non-emergencies (priority 4 ).

Inclusion criteria were patients who presented to the ED with age greater than 16 years or $>40 \mathrm{~kg}$ body weight requiring intravenous fluids or medications and in whom an intravenous line could not be established in two attempts or $90 \mathrm{~s}$. They also had to be seriously ill or injured and possess one or more of the following:

1. An altered mental state [score on the Glasgow Coma Scale (GCS) of 8 or less]

2. Respiratory compromise [oxygen saturation $\left(\mathrm{SaO}_{2}\right)$ $80 \%$ after appropriate oxygen therapy, respiratory rate $<10$ or $>40 / \mathrm{min}$ ]

3. Haemodynamic instability [systolic blood pressure (BP) of <90] or profound hypovolaemia (signs and symptoms of shock)

4. Cardiac arrest (medical or traumatic)

Insertion of an IO needle was contraindicated if there was a fracture of the tibia or femur, recent surgery in the extremity to be used or previous orthopaedic procedure (knee replacement) or IO within $24 \mathrm{~h}$ (consider alternate tibia), pre-existing medical condition (tumour near insertion site or peripheral vascular disease), infection at insertion site or significant oedema in the extremity to be used. The use of the alternate tibia would be considered in those scenarios. Inability to locate landmarks, significant oedema and excessive tissue at insertion site were relative contraindications to insertion.

The EZ-IO ${ }^{\mathrm{TM}}$ utilizes a reusable battery-powered driver and a disposable IO needle set that powers into the IO space by rotating a hollow drill to a preset depth $\left(\mathrm{EZ}^{\mathrm{I}} \mathrm{IO}{ }^{\mathrm{TM}}\right.$ System Driver model number 9050, PD needle set model 9018, AD needle set model 9001, Vidacare Corporation, San Antonio, TX, USA).

Sites of insertion included the tibia and the humerus. The landmark for the insertion point of the proximal tibia is 2 fingerbreadths below the patella and $1-2 \mathrm{~cm}$ medial to the tibial tuberosity. The landmark for the insertion point for the proximal humerus is the most prominent aspect of the greater tubercle's outer margins, with the patient's hand on his abdomen to ensure the safest position. A pressure bag device would be applied to the infusion if its rate of infusion was deemed to be too slow.

The outcomes that were assessed included the success rate of EZ-IO ${ }^{\mathrm{TM}}$ insertion by operators as defined by the ability to place the IO needle, as well successfully infusing fluids and drugs. Flow rates using normal saline infusion were measured by an independent nurse observer. Methods of IO needle placement confirmation, estimated total time of insertion, fluids and drugs administered, ease of use and the control and function of the EZ-IO ${ }^{\mathrm{TM}}$ device, any complications, difficulties in using the device, adverse events to operator and types of malfunction of device were evaluated by a questionnaire.

Placement time was recorded by the individual operator, recorded as the time the operator placed the needle set into the driver till the time the needle was successfully inserted into the bone. The difficulty of insertion was recorded by the physicians on a $10-\mathrm{cm}$ visual analogue scale (VAS) with 0 representing very easy placement and 10 representing very difficult placement.

Only emergency physicians and medical residents who were trained in the use of the EZ-IO ${ }^{\mathrm{TM}}$ by completing the manufacturer's training programme and who were familiar with the protocol were allowed to use the device. Standard training for IO insertion was straightforward and lasted less than $2 \mathrm{~h}$. This consisted of a lecture and hands-on training $[20,21]$. The physicians were given instructions on the use of the EZ-IO ${ }^{\mathrm{TM}}$ and observed a demonstration on its use on a standard plastic bone model of the tibia as provided for by the manufacturer. The physicians were subsequently allowed multiple practices to obtain IO access using the EZ-IO ${ }^{\mathrm{TM}}$ on the plastic bone model.

During the actual IO placement, needle placement was confirmed by visualization of blood on the stylet, ability to aspirate bone marrow, firm placement of the needle in the bone and ability to smoothly deliver a fluid flush. Operators were instructed to give a rapid flush (bolus) of $10 \mathrm{ml}$ of saline with a syringe through the EZ-IO ${ }^{\mathrm{TM}}$ once needle placement was confirmed. For conscious patients, a prior flush (bolus) of 20-50 mg 2\% lidocaine (preservative free) through the EZ$\mathrm{IO}^{\mathrm{TM}}$ was recommended for local anaesthesia.

Patients were followed up until hospital discharge for any complications of IO insertion, including needle displacement, failure of the drill device to function properly, fractures, infection of the insertion site, osteomyelitis, fat embolism, extravasation of fluid or medication and compartment syndrome.

Data were entered using Microsoft Excel 2002 (version 10) and data analysis was performed using SPSS Statistics 15.0. 
Descriptive frequencies and means/standard deviations are reported as appropriate. Fischer's exact test was used for categorical comparisons and the Mann-Whitney U test for equivalent non-parametric comparisons.

\section{Results}

From 1 March 2006 to 30 July 2007, 24 patients were recruited. The tibia was the first site of insertion, and a second IO was inserted in the humerus if clinically indicated for the same patient. Although there were 24 patients, there were 24 tibial insertions and 11 humeral insertions on the same patient where clinically indicated for a second peripheral access. The characteristics of the patients are listed in Table 1.

All EZ-IOTM insertions were successful at the first attempt except for three tibial insertions that were successful on the second attempt. All insertions were firmly placed, with good control of the needle set and separation from the driver.

Table 2 shows a comparison between junior operators (residents) and senior operators (attendings). Both groups had $100 \%$ success rates for insertion. The average insertion time was less than $5 \mathrm{~s}$ for both groups. In our study, the VAS was 1.06 . We noted that $88.6 \%$ of physicians in our study reported easier insertion with the $\mathrm{EZ}-\mathrm{IO}^{\mathrm{TM}}$ as compared to an intravenous cannula.

The junior operators required more repeated attempts and encountered low flow rates as compared to the senior operators.

There were two complications encountered by a senior operator. A glove was caught in the drill device during the IO insertion. The physician had held the IO needle to stabilize the needle's position on the patient's tibia while applying the drill. As such, the glove was caught in the needle and wound around the IO needle as the drill was

Table 1 EZ-IO: characteristics of study participants

\begin{tabular}{lll}
\hline & $N=24$ & $\%$ \\
\hline Age 16-29 years & 2 & 8.3 \\
Age 30-59 years & 6 & 25 \\
Age $>$ 60 years & 16 & 66.7 \\
Race & & \\
Chinese & 17 & 70.8 \\
Indian & 4 & 16.7 \\
Malay/others & 3 & 12.5 \\
Male & 12 & 50 \\
GCS 8-15 & 8 & 33.3 \\
GCS 3-7 & 16 & 66.7 \\
Trauma & 4 & 16.7 \\
\hline
\end{tabular}

Table 2 Comparison of intraosseous insertions between operators

\begin{tabular}{llll}
\hline & $\begin{array}{l}\text { Resident, } \\
N=8(\%)\end{array}$ & $\begin{array}{l}\text { Attending/ } \\
\text { consultant, } \\
N=27(\%)\end{array}$ & $P$ value \\
& & $27(100.0)$ & 1 \\
\hline EZ-IO placed successfully (\%) & $8(100.0)$ & $27(3.7)$ & 0.083 \\
Multiple attempts needed (\%) & $2(25)$ & 1.0 & 0.920 \\
Visual analogue scale (0-10) & 1.1 & 1.0 & 1 \\
EZ-IO firmly placed (\%) & $8(100.0)$ & $27(100.0)$ & 1 \\
Good control of needle set (\%) & $8(100.0)$ & $27(100.0)$ & 1 \\
Needle separated from driver & $8(100.0)$ & $27(100.0)$ & 1 \\
$\quad$ easily (\%) & $8(100.0)$ & $27(100.0)$ & 1 \\
Stylet separated from needle & & & \\
$\quad$ easily (\%) & $8(100.0)$ & $23(85.2)$ & 0.553 \\
Easier placement with the EZ-IO & & & 1 \\
$\quad$ than an intravenous cannula (\%) & $1(12.5)$ & 0 & 0.030 \\
No flow initially & $3(37.5)$ & $1(2.9)$ & 1 \\
Low flow rates & 0 & $2(5.7)$ & 0.862 \\
$\begin{array}{l}\text { Complications } \\
\text { Insertion time (average), s }\end{array}$ & 3.9 & 4.3 & \\
\hline
\end{tabular}

applied. Another complication encountered was extravasation of infusion fluid at the site of insertion of the IO needle.

Table 3 shows the flow rates of intraosseous infusions with and without pressure bag application. Flow rates were significantly faster with a pressure bag than without. Tibial flow rates were $204.6 \mathrm{ml} / \mathrm{min}$ with a pressure bag as compared to $68.2 \mathrm{ml} / \mathrm{min}$ without a pressure bag, difference $-129.5 \mathrm{ml} / \mathrm{min}[95 \%$ confidence interval $(\mathrm{CI}):-218.2$ to $-40.3)$. Humeral flow rates were significantly faster using a pressure bag $(148.1 \mathrm{ml} / \mathrm{min})$ as compared to without $(81.8 \mathrm{ml} / \mathrm{min})$, difference $-69.6 \mathrm{ml} / \mathrm{min}(95 \% \mathrm{CI}:-113.9$ to -25.3 ). But the difference of changes ( with or without pressure bag) of flow rate between the tibia and humerus did not show any significance $(P=0.157$, Mann-Whitney test).

There were no complications of needle displacement, failure of the drill device to function properly, fractures, infection of the insertion site, osteomyelitis, fat embolism or compartment syndrome.

\section{Discussion}

Available IO devices have included manual needles including the Cook device and the Jamshidi needles, spring-loaded "punch devices" including the First Access for Shock and Trauma (F.A.S.T., Pyng) IO device in the sternum [22] and the Bone Injection Gun (B.I.G) in the tibia [23], which has been extensively used by the Israeli military [24].

Preparation and insertion for the Pyng approaches $50 \mathrm{~s}$ [22] with success rates of $74 \%$ for first time users and $95 \%$ 
Table 3 Comparison of flow rates with/without pressure bag

$P=0.157$ (Mann-Whitney test)

\begin{tabular}{lll}
\hline Flow rate & Tibia $(N=10), \mathrm{ml} / \mathrm{min}$ & Humerus $(N=8), \mathrm{ml} / \mathrm{min}$ \\
\hline No pressure bag & $68.2(42.1)$ & $81.8(38.4)$ \\
With pressure bag & $204.6(156.0)$ & $148.1(75.3)$ \\
Difference & -129.5 & -69.6 \\
$95 \% \mathrm{CI}$ & -218.2 to -40.3 & -113.9 to -25.3 \\
\hline
\end{tabular}

for experienced users. This may be particularly useful for patients with lower extremity or pelvic trauma [25]. The B. I.G. is reported to require about $17 \mathrm{~s}$ for preparation and insertion [26]. Preparation and insertion time for the EZ-IO is reported to be approximately $10 \mathrm{~s}$ [27]. Prehospital trials on the EZ-IO device show an $87 \%$ success rate. The F.A.S. T.1 device used in the same trial showed $72 \%$ success [28].

In our hospital, the usual practice was to attempt multiple peripheral IV insertions even though access may be difficult or impossible. Central venous access is usually not attempted, as it takes too long as compared to the intraosseous method.

In our study, we had $100 \%$ success rates for both junior and senior operators. Intraosseous flow rates were significantly faster with a pressure bag infusion than without.

The IO needle had previously been shown to be a rapid and effective method of vascular access [3, 4, 11, 13, 16, 19]. We found that even previously inexperienced operators could rapidly and safely insert the IO needle. Previous studies have also shown that emergency drugs and fluids can be rapidly delivered to the systemic circulation, at rates comparable to the intravenous and central venous routes [28, 29]. All medications and blood products that are approved for IV infusion can be given via an IO access. In our study, the fluids and drugs used included normal saline, dextrose, calcium chloride, atropine, epinephrine, frusemide and ceftriaxone.

The maximum rate of administration through the IO needle was reportedly equivalent to a $21 \mathrm{G}$ peripheral cannula [23]. The flow rates of an intravenous cannula are typically in the range of 200 (16 G peripheral cannula) to $20 \mathrm{ml} / \mathrm{min}$ (24 G peripheral cannula) [30]. EZ-IO ${ }^{\mathrm{TM}}$ tibial flow rates ranged from 20 to over $3,000 \mathrm{ml} / \mathrm{h}$ under pressure [27].

In our study, we found that use of a pressure infusion bag significantly improved fluid flow rates via the IO needle. This is similar to a previous report using paediatric IO needles [31], although we were able to achieve higher flow rates using adult $\mathrm{IO}$ needles, hence subjectively able to provide the necessary volume resuscitation in such circumstances. Pressure pumps improve flow of the IO needle. Flushing of the IO needle after insertion seems to improve flow rates as well [27].

Various site have been proposed as suitable for IO insertions, including the proximal tibia [3, 4, 13], distal tibia [2], sternum [4, 12, 13, 22], radius [32], clavicle [14], proximal humerus and calcaneum [33]. The proximal tibia and proximal humerus sites were chosen for this study. The proximal tibia site was the initial insertion site of choice, as the landmarks were easily identifiable, superficial, easy to access percutaneously and proximal enough to allow rapid access of fluids or medications into the central circulation. In addition, it is away from vital areas where other resuscitation procedures are ongoing as well as vital structures that might get inadvertently punctured during insertion. For example, the sternal and clavicular sites present problems when airway procedures and cervical immobilization are ongoing in trauma resuscitation. Likewise, the investigators felt that the distal tibia, radius and calcaneum sites would be relatively distal to the central circulation. The proximal humerus was the secondary site, in the event that intravenous cannulation was still unsuccessful after initial resuscitation.

Previously reported complications associated with IO insertions include osteomyelitis [34], extravasation [35], fat embolism [36], compartment syndrome [37], growth plate abnormalities [38] and myonecrosis with hypertonic saline infusion [39]. There were no reported complications with the device in this study, except for two cases. One was where the operator's glove was caught in the IO drill device during insertion. This could be easily prevented by not holding the IO needle during the insertion process but just allowing the drill to insert the IO needle.

Another complication was that of extravasation of fluid at the site of insertion. Extravasation of fluid is the most common complication [40]. It typically occurs when a needle is misplaced. It seldom occurs with a properly placed needle, and it is associated with excessive movement during or after insertion, which may lead to enlargement of the entry site in the bone relative to the diameter of the needle. Compartment syndrome is a risk with extravasation although our patient did not develop compartment syndrome. The needle must enter through the cortex and into the marrow cavity without passing through the cortex on the other side. If the needle is passed through the opposite cortex, infused fluid enters the calf rather than the venous system. Fluid accumulation may lead to a compartment syndrome. This complication can also be limited by making frequent checks and allowing only one attempt per tibia. Repeated attempts in the same bone allow fluid to flow 
through the previous holes produced in the bone. Extravasation of hypertonic or caustic medications, such as sodium bicarbonate, dopamine or calcium chloride, can result in necrosis of the muscle.

There were two more cases where ward staff reported difficulty removing the needle. However, the needles were both successfully removed once the correct technique was applied. The correct technique involves using a rotating and pulling movement, rather than rocking the needle, which may cause it to break. Also the needle comes with a Luer lock which can be attached to a syringe to use as a handle for additional traction during removal.

Limitations of the study include the relatively small sample size. In many cases, subsequent intravenous cannulation was successful, once initial resuscitation had been initiated through the tibial IO access.

Also the insertion times were not recorded by an independent observer due to the logistic difficulty of having an investigator present at every resuscitation. We also noted that insertion times may be longer in a true clinical setting, as some time was required to assemble the driver and needle. However, this time is minimal compared to the actual insertion procedure.

The EZ-IO ${ }^{\mathrm{TM}}$ allowed medical personnel with little prior experience of adult IO access to be able to achieve successful placement in a fast mean. We also recommend routine use of a pressure infusion bag in order to improve flow rates.

\section{Conclusion}

The EZ-IOTM is a feasible, useful and fast alternative mode of venous access especially in the resuscitation of patients with no venous access or when conventional intravenous access fails. Flow rates may be improved by the use of pressure bags. Complications encountered such as extravasation of fluid and gloves being caught in the drill device can be easily prevented.

Acknowledgement We acknowledge the support of Vidacare Corporation, San Antonio, TX, USA, in providing the EZ-IO devices used in the study. No cast sponsorship was used for this study.

Conflicts of interest The authors declare that they have no other conflict of interest or disclosures.

\section{References}

1. Drinker CK, Drinker KR, Lund CC (1922) The circulation in the mammalian bone marrow. Am J Physiol 62:1-92

2. Morrison GM (1946) The initial care of casualties. Am Pract $1: 183-184$

3. Boon JM, Gorry DL, Meiring JH (2003) Finding an ideal site for intraosseous infusion of the tibia: an anatomical study. Clin Anat $16: 15-18$
4. Frascone RJ, Jensen JP, Kaye K, Salzman JG (2007) Consecutive field trials using two different intraosseous devices. Prehosp Emerg Care 11:164-171

5. Mattera CJ (2000) Take aim-hit your IO target. A comprehensive approach to pediatric intraosseous infusion, including site selection, needle insertion \& ongoing assessment. Part 2. JEMS 25:3848

6. Cilley RE (1992) Intraosseous infusion in infants and children. Semin Pediatr Surg 1:202-207

7. Helm M, Hauke J, Bippus N, Lampl L (2007) Intraosseous puncture in preclinical emergency medicine. Ten years experience in air rescue service (in German). Anaesthesist 56:18-24

8. Jaimovich DG, Kecskes S (1991) Intraosseous infusion: a rediscovered procedure as an alternative for pediatric vascular access. Indian J Pediatr 58:329-334

9. Seigler RS, Tecklenburg FW, Shealy R (1989) Prehospital intraosseous infusion by emergency medical services personnel: a prospective study. Pediatrics 84:173-177

10. Velasco AL, Delgado-Paredes C, Templeton J, Steigman CK, Templeton JM Jr (1991) Intraosseous infusion of fluids in the initial management of hypovolemic shock in young subjects. J Pediatr Surg 26:4-8

11. Wagner MB, McCabe JB (1988) A comparison of four techniques to establish intraosseous infusion. Pediatr Emerg Care 4:87-91

12. Calkins MD, Fitzgerald G, Bentley TB, Burris D (2000) Intraosseous infusion devices: a comparison for potential use in special operations. J Trauma 48:1068-1074

13. Glaeser PW, Hellmich TR, Szewczuga D, Losek JD, Smith DS (1993) Five-year experience in prehospital intraosseous infusions in children and adults. Ann Emerg Med 22:1119-1124

14. Iwama H, Katsumi A, Shinohara K, Kawamae K, Ohtomo Y, Akama Y, Tase C, Okuaki A (1994) Clavicular approach to intraosseous infusion in adults. Fukushima J Med Sci 40:1-8

15. LaRocco BG, Wang HE (2003) Intraosseous infusion. Prehosp Emerg Care 7:280-285

16. Timboe HL, Bruttig SP, Ruemmler MW (2005) Adult IO in the combat zone: the past, present and future use of intraosseous infusion by the U.S. military. JEMS 30(10):suppl 27-suppl 28

17. Fernandes CM (1991) Intraosseus needles. Arch Emerg Med 8:68-71

18. Glaeser PW, Losek JD (1988) Intraosseous needles: new and improved. Pediatr Emerg Care 4:135-136

19. Jun H, Haruyama AZ, Chang KS, Yamamoto LG (2000) Comparison of a new screw-tipped intraosseous needle versus a standard bone marrow aspiration needle for infusion. Am J Emerg Med 18:135-139

20. Anderson TE, Arthur K, Kleinman M et al (1994) Intraosseous infusion: success of a standardized regional training program for prehospital advanced life support providers. Ann Emerg Med 23 (1):52-55

21. Dubick MA, Holcomb JB (2000) A review of intraosseous vascular access: current status and military application. Mil Med 165(7):552-559

22. Miller DD, Guimond G, Hostler DP et al (2005) Feasibility of sternal intraosseous access by emergency medical technician students. Prehosp Emerg Care 9(1):73-78

23. Miller L, Kramer GC, Bolleter S (2005) Rescue access made easy. JEMS 30(10):suppl 8-suppl 18

24. Curran A, Sen A (2005) Bone injection gun placement of intraosseous needles. Emerg Med J 22(5):366

25. Macnab A, Christenson J, Findlay J, Horwood B, Johnson D, Jones L et al (2000) A new system for sternal intraosseous infusion in adults. Prehosp Emerg Care 4(2):173-177

26. Spriggs NM, White LJ, Martin SW et al (2000) Comparison of two intraosseous infusion techniques in an EMT training program. Acad Emerg Med 7(10):1168 
27. Davidoff J, Fowler R, Gordon D et al (2005) Clinical evaluation of a novel intraosseous device for adults: prospective, 250-patient, multi-center trial. JEMS 30(10):suppl 20-suppl 23

28. Neufeld JD, Marx JA, Moore EE, Light AI (1993) Comparison of intraosseous, central, and peripheral routes of crystalloid infusion for resuscitation of hemorrhagic shock in a swine model. J Trauma $34: 422-428$

29. Orlowski JP, Porembka DT, Gallagher JM, Lockrem JD, VanLente F (1990) Comparison study of intraosseous, central intravenous, and peripheral intravenous infusions of emergency drugs. Am J Dis Child 144:112-117

30. Jayanthi NV, Dabke HV (2006) The effect of IV cannula length on the rate of infusion. Injury $37(1): 41-45$

31. Hodge D 3rd, Delgado-Paredes C, Fleisher G (1987) Intraosseous infusion flow rates in hypovolemic "pediatric" dogs. Ann Emerg Med 16:305-307

32. Waisman M, Waisman D (1997) Bone marrow infusion in adults. J Trauma 42:288-293

33. McCarthy G, Buss P (1998) The calcaneum as a site for intraosseous infusion. J Accid Emerg Med 15:421

34. Stoll E, Golej J, Burda G, Hermon M, Boigner H, Trittenwein G (2002) Osteomyelitis at the injection site of adrenalin through an intraosseous needle in a 3-month-old infant. Resuscitation 53:315318

35. LaSpada J, Kissoon N, Melker R, Murphy S, Miller G, Peterson R (1995) Extravasation rates and complications of intraosseous needles during gravity and pressure infusion. Crit Care Med 23:2023-2028

36. Hasan MY, Kissoon N, Khan TM, Saldajeno V, Goldstein J, Murphy SP (2001) Intraosseous infusion and pulmonary fat embolism. Pediatr Crit Care Med 2:133-138

37. Günal I, Köse N, Gürer D (1996) Compartment syndrome after intraosseous infusion: an experimental study in dogs. J Pediatr Surg 31:1491-1493

38. Brickman KR, Rega P, Koltz M, Guinness M (1988) Analysis of growth plate abnormalities following intraosseous infusion through the proximal tibial epiphysis in pigs. Ann Emerg Med 17:121-123

39. Alam HB, Punzalan CM, Koustova E, Bowyer MW, Rhee P (2002) Hypertonic saline: intraosseous infusion causes myonecrosis in a dehydrated swine model of uncontrolled hemorrhagic shock. J Trauma 52:18-25

40. Gluckman W (2008) Intraosseous cannulation. Emedicine. http:// www.emedicine.com/ped/TOPIC2557.HTM. Accessed 17 Aug 2008

Adeline Su-Yin Ngo is a consultant with the Department of Emergency Medicine, Singapore General Hospital, Singapore. She is also a Fellow of the Academy of Medicine (Emergency Medicine), a clinical teacher at the School of Medicine, National University of Singapore, an instructor for advanced cardiac life support, Singapore, automated electrical defibrillation, Singapore as well as an instructor for Hazmat medical life support.

Jen Jen Oh is an Associate Consultant with the Department of Emergency Medicine, Singapore General Hospital, Singapore.

Yuming Chen works at the Singapore Clinical Research Institute. He does research and is a consultant for statistics.

David Yong is a research assistant at the Department of Emergency Medicine, Singapore General Hospital, Singapore.

Marcus Eng Hock Ong is a Consultant with the Department of Emergency Medicine, Singapore General Hospital, Singapore. He is a Fellow of the Academy of Medicine (Emergency Medicine), a clinical teacher at the School of Medicine, National University of Singapore, and an instructor for advanced cardiac life support, Singapore. 